\title{
Two-dimensional hydrogen-like atom in a weak magnetic field
}

\author{
Radosław Szmytkowski ${ }^{\mathrm{a}}$
}

Atomic and Optical Physics Division, Department of Atomic, Molecular and Optical Physics, Faculty of Applied Physics and Mathematics, Gdańsk University of Technology, ul. Gabriela Narutowicza 11/12, 80-233 Gdańsk, Poland

Received: 7 June 2018 / Revised: 19 June 2018

Published online: 6 August 2018

(C) The Author(s) 2018. This article is published with open access at Springerlink.com

\begin{abstract}
We consider a non-relativistic two-dimensional (2D) hydrogen-like atom in a weak, static, uniform magnetic field perpendicular to the atomic plane. Within the framework of the Rayleigh-Schrödinger perturbation theory, using the Sturmian expansion of the generalized radial Coulomb Green function, we derive explicit analytical expressions for corrections to an arbitrary planar hydrogenic bound-state energy level, up to the fourth order in the strength of the perturbing magnetic field. In the case of the ground state, we correct an expression for the fourth-order correction to energy available in the literature.
\end{abstract}

\section{Introduction}

Theoretical studies on elementary two-dimensional quantum structures in magnetic fields have been carried out for several decades [1-20]. Recent spectacular developments in single-layer materials science have given a fresh impact to such investigations [21-36]. As a result of the work done so far, at the present moment we understand some aspects of the magnetic-field-induced properties of $2 \mathrm{D}$ analogues of atoms and molecules, but our knowledge on the subject still appears to be far from being complete.

Recently, we have come across the need to know exact analytical representations for low-order perturbation theory corrections to an arbitrary energy level of a two-dimensional analogue of a hydrogen-like atom placed in a weak and uniform magnetic field perpendicular to the atomic plane. The first-order correction may be obtained trivially for any atomic state. Exact values of the second-order corrections for states with the principal quantum numbers $1 \leqslant n \leqslant 4$ may be derived from a table provided in ref. [4]. The third-order correction may be shown to vanish identically for any state (in fact, the same happens for all odd-order corrections other than the first-order one), while in refs. [25,31] the fourth-order correction has been given, but for the ground level only. Approximate expressions for several higher even-order corrections to states with zero radial quantum numbers and with principal quantum numbers not exceeding six are contained in ref. [35]. However, neither of the publications invoked above, nor any other related one we have had in hands in the course of browsing the literature, contains the general formulas we have been seeking for. This is a bit astonishing in view of the fact that for a similar problem of the planar one-electron atom placed in a weak, uniform, in-plane electric field, closed-form analytical expressions for Stark-Lo Surdo corrections to energies of discrete parabolic eigenstates are known up to the sixth order in the perturbing field $[37,38]$. Under these circumstances, we have derived expressions for the second- and fourth-order magnetic-field-induced corrections to an arbitrary energy level of the planar hydrogenic atom. The results of that study are presented in this work. We believe they may be of some interest, in particular because the result for the fourth-order correction to the ground state given in ref. [25], and then repeated in ref. [31], has been found to be incorrect.

\section{Preliminaries}

We consider a one-electron atom with a point-like and spinless nucleus at rest. The electron is constrained to move in a plane through the nucleus. A potential of interaction between the nucleus (of charge $+Z e$ ) and the electron (of charge $-e$ and mass $m$ ) is taken to be the attractive Coulomb one-over-distance one. The system is subjected to the action of

\footnotetext{
a e-mail: radoslaw.szmytkowski@pg.edu.pl
} 
a static uniform magnetic field of induction $\boldsymbol{B}$, which is perpendicular to the atomic plane. With the electron radius vector $\boldsymbol{r}$ being referred to the nucleus, the two-dimensional time-independent Schrödinger equation for the electron is

$$
\left\{\frac{[-\mathrm{i} \hbar \boldsymbol{\nabla}+e \boldsymbol{A}(\boldsymbol{r})]^{2}}{2 m}-\frac{Z e^{2}}{\left(4 \pi \epsilon_{0}\right) r}\right\} \Psi(\boldsymbol{r})=E \Psi(\boldsymbol{r}) \quad\left(\boldsymbol{r} \in \mathbb{R}^{2}\right),
$$

where $r=|\boldsymbol{r}|$ and $\boldsymbol{A}(\boldsymbol{r})$ is a vector potential of the magnetic field. Equation (1) is to be solved, with the electron energy $E$ chosen as an eigenvalue, subject to the constraint that the wave function $\Psi(\boldsymbol{r})$ is single-valued and bounded for all $\boldsymbol{r} \in \mathbb{R}^{2}$, including the point $\boldsymbol{r}=0$ and the point at infinity.

Throughout this paper, we shall be working in the symmetric gauge, in which the vector potential $\boldsymbol{A}(\boldsymbol{r})$ is

$$
\boldsymbol{A}(\boldsymbol{r})=\frac{1}{2} \boldsymbol{B} \times \boldsymbol{r}
$$

Then, the Schrödinger equation (1) may be rewritten as

$$
\left[-\frac{\hbar^{2}}{2 m} \nabla^{2}+\frac{e \hbar}{2 m} \boldsymbol{B} \cdot \boldsymbol{\Lambda}+\frac{e^{2} B^{2} r^{2}}{8 m}-\frac{Z e^{2}}{\left(4 \pi \epsilon_{0}\right) r}\right] \Psi(\boldsymbol{r})=E \Psi(\boldsymbol{r}),
$$

where

$$
\boldsymbol{\Lambda}=-\mathrm{i} \boldsymbol{r} \times \boldsymbol{\nabla}
$$

is a (dimensionless) orbital angular-momentum operator for the electron. The form of the Hamiltonian operator in the Schrödinger equation (3) suggests one introduces the polar coordinates $r$ and $\varphi$, with $0 \leqslant r<\infty$ and $0 \leqslant \varphi<2 \pi$; eq. (3) is then transformed into the following one:

$$
\left[-\frac{\hbar^{2}}{2 m}\left(\frac{\partial^{2}}{\partial r^{2}}+\frac{1}{r} \frac{\partial}{\partial r}+\frac{1}{r^{2}} \frac{\partial^{2}}{\partial \varphi^{2}}\right)-\frac{\mathrm{i} e \hbar B}{2 m} \frac{\partial}{\partial \varphi}+\frac{e^{2} B^{2} r^{2}}{8 m}-\frac{Z e^{2}}{\left(4 \pi \epsilon_{0}\right) r}\right] \Psi(r, \varphi)=E \Psi(r, \varphi) .
$$

The benefit from the use of the polar coordinates is that eq. (5) is separable, in the sense that it possesses particular solutions of the form

$$
\Psi_{n l m_{l}}(r, \varphi)=\frac{1}{\sqrt{r}} P_{n l m_{l}}(r) \Phi_{m_{l}}(\varphi) \quad\left(l=\left|m_{l}\right|\right),
$$

where

$$
\Phi_{m_{l}}(\varphi)=\frac{1}{\sqrt{2 \pi}} \mathrm{e}^{\mathrm{i} m_{l} \varphi} \quad\left(m_{l} \in \mathbb{Z}\right) .
$$

Plugging eq. (6) into eq. (5) and exploiting eq. (7) yields the radial Schrödinger equation

$$
\left[-\frac{\hbar^{2}}{2 m} \frac{\mathrm{d}^{2}}{\mathrm{~d} r^{2}}+\frac{\hbar^{2}\left(l^{2}-\frac{1}{4}\right)}{2 m r^{2}}+m_{l} \frac{e \hbar B}{2 m}+\frac{e^{2} B^{2} r^{2}}{8 m}-\frac{Z e^{2}}{\left(4 \pi \epsilon_{0}\right) r}\right] P_{n l m_{l}}(r)=E_{n l m_{l}} P_{n l m_{l}}(r),
$$

which is to be solved subject to the boundary conditions

$$
P_{n l m_{l}}(r) / \sqrt{r} \quad \text { bounded for } r \rightarrow 0 \text { and for } r \rightarrow \infty \text {. }
$$

It is easy to deduce from the standard asymptotic analysis that for $B \neq 0$ the constraints displayed in eq. (8b) may be replaced by the following ones:

$$
P_{n l m_{l}}(r) \stackrel{r \rightarrow 0}{\longrightarrow} 0, \quad P_{n l m_{l}}(r) \stackrel{r \rightarrow \infty}{\longrightarrow} 0 .
$$

The symbol $n$ that has appeared the first time as a subscript in eq. (6) is the principal quantum number defined as

$$
n=n_{r}+l+1
$$

where $n_{r} \in \mathbb{N}_{0}$ is the radial quantum number which counts the number of nodes (zeroes) in the radial wave function.

Since the term linear in $B$ which appears in the differential operator in eq. (8a) is independent of the variable $r$, it is clear that the energy eigenvalue $E_{n l m_{l}}$ may be written as

$$
E_{n l m_{l}}=E_{n l}+E_{m_{l}}^{(1)}
$$

with

$$
E_{m_{l}}^{(1)}=m_{l} \frac{e \hbar B}{2 m} .
$$


It is also evident that the radial function $P_{n l m_{l}}(r)$ does depend on $m_{l}$ through $l=\left|m_{l}\right|$ only:

$$
P_{n l m_{l}}(r) \equiv P_{n l}(r)
$$

Consequently, the starting point for further considerations will be the radial eigenvalue problem

$$
\begin{aligned}
& {\left[-\frac{\hbar^{2}}{2 m} \frac{\mathrm{d}^{2}}{\mathrm{~d} r^{2}}+\frac{\hbar^{2}\left(l^{2}-\frac{1}{4}\right)}{2 m r^{2}}-\frac{Z e^{2}}{\left(4 \pi \epsilon_{0}\right) r}+\frac{e^{2} B^{2} r^{2}}{8 m}\right] P_{n l}(r)=E_{n l} P_{n l}(r),} \\
& P_{n l}(r) \stackrel{r \rightarrow 0}{\longrightarrow} 0, \quad P_{n l}(r) \stackrel{r \rightarrow \infty}{\longrightarrow} 0 .
\end{aligned}
$$

\section{Perturbation theory analysis}

\subsection{Basics and the zeroth-order problem}

Closed-form analytical solutions to the eigenproblem (13) are not known. Therefore, below we shall attempt to find its approximate solutions, under the assumption that the magnetic field is weak, with the use of the Rayleigh-Schrödinger perturbation theory. To this end, we write the radial differential operator from eq. (13a) as

$$
H_{l}(r)=H_{l}^{(0)}(r)+H^{(2)}(r)
$$

where

$$
H_{l}^{(0)}(r)=-\frac{\hbar^{2}}{2 m} \frac{\mathrm{d}^{2}}{\mathrm{~d} r^{2}}+\frac{\hbar^{2}\left(l^{2}-\frac{1}{4}\right)}{2 m r^{2}}-\frac{Z e^{2}}{\left(4 \pi \epsilon_{0}\right) r}
$$

and

$$
H^{(2)}(r)=\frac{e^{2} B^{2} r^{2}}{8 m}
$$

We shall treat the diamagnetic term (16) as a small perturbation of the radial Coulomb Hamiltonian (15). Since $H^{(2)}(r)$ is of the second order in the perturbing magnetic field, we seek solutions to the eigensystem (13) in the form of the perturbation series

$$
E_{n l}=E_{n l}^{(0)}+E_{n l}^{(2)}+E_{n l}^{(4)}+\cdots
$$

and

$$
P_{n l}(r)=P_{n l}^{(0)}(r)+P_{n l}^{(2)}(r)+P_{n l}^{(4)}(r)+\cdots,
$$

involving even-order terms only. Here $E_{n l}^{(0)}$ and $P_{n l}^{(0)}(r)$ are those solutions to the zeroth-order eigenproblem (being the radial Coulomb one)

$$
\begin{aligned}
& {\left[H_{l}^{(0)}(r)-E^{(0)}\right] P^{(0)}(r)=0,} \\
& P^{(0)}(r) \stackrel{r \rightarrow 0}{\longrightarrow} 0, \quad P^{(0)}(r) \text { bounded for } r \rightarrow \infty
\end{aligned}
$$

(subscripts have been omitted intentionally), which correspond to the discrete part of its spectrum, consisting of the eigenvalues

$$
E_{n l}^{(0)} \equiv E_{n}^{(0)}=-\frac{Z^{2}}{2 N_{n}^{2}} \frac{e^{2}}{\left(4 \pi \epsilon_{0}\right) a_{0}},
$$

with

$$
N_{n}=n-\frac{1}{2}=n_{r}+l+\frac{1}{2}
$$

and with

$$
a_{0}=\left(4 \pi \epsilon_{0}\right) \frac{\hbar^{2}}{m e^{2}}
$$

being the Bohr radius. Eigenfunctions associated with the eigenvalues (20), orthonormal in the sense of

$$
\int_{0}^{\infty} \mathrm{d} r P_{n l}^{(0)}(r) P_{n^{\prime} l}^{(0)}(r)=\delta_{n n^{\prime}}
$$


are

$$
P_{n l}^{(0)}(r)=\sqrt{\frac{Z(n-l-1) !}{a_{0} N_{n}^{2}(n+l-1) !}}\left(\frac{2 Z r}{N_{n} a_{0}}\right)^{l+1 / 2} \mathrm{e}^{-Z r / N_{n} a_{0}} L_{n-l-1}^{(2 l)}\left(\frac{2 Z r}{N_{n} a_{0}}\right),
$$

where $L_{k}^{(\alpha)}(x)$ is the generalized Laguerre polynomial ([39], sect. 5.5). For integration purposes, it is frequently convenient to have these functions rewritten as

$$
P_{n l}^{(0)}(r)=\sqrt{\frac{Z n_{r} !}{a_{0} N_{n}^{2}\left(n_{r}+2 l\right) !}}\left(\frac{2 Z r}{N_{n} a_{0}}\right)^{l+1 / 2} \mathrm{e}^{-Z r / N_{n} a_{0}} L_{n_{r}}^{(2 l)}\left(\frac{2 Z r}{N_{n} a_{0}}\right) .
$$

\subsection{The second-order corrections to Coulomb energies}

For the present problem, the second-order correction to energy, $E_{n l}^{(2)}$, is given by

$$
E_{n l}^{(2)}=\int_{0}^{\infty} \mathrm{d} r P_{n l}^{(0)}(r) H^{(2)}(r) P_{n l}^{(0)}(r)
$$

or, equivalently, if use is made of eq. (16), by

$$
E_{n l}^{(2)}=\frac{e^{2} B^{2}}{8 m} \int_{0}^{\infty} \mathrm{d} r r^{2}\left[P_{n l}^{(0)}(r)\right]^{2}
$$

Plugging eq. (25) into the integrand and exploiting the integration formula

$$
\begin{aligned}
& \int_{0}^{\infty} \mathrm{d} x x^{\alpha+3} \mathrm{e}^{-x}\left[L_{k}^{(\alpha)}(x)\right]^{2}=(2 k+\alpha+1)\left(10 k^{2}+10 k+10 \alpha k+\alpha^{2}+5 \alpha+6\right) \frac{\Gamma(k+\alpha+1)}{k !} \\
& (\operatorname{Re} \alpha>-4),
\end{aligned}
$$

which may be deduced from the general expression ([40], eqs. (E54), (E56) and (E60))

$$
\begin{aligned}
& \int_{0}^{\infty} \mathrm{d} x x^{\gamma} \mathrm{e}^{-x} L_{k}^{(\alpha)}(x) L_{k^{\prime}}^{(\beta)}(x)=(-)^{k+k^{\prime}} \sum_{m=0}^{\min \left(k, k^{\prime}\right)} \frac{\Gamma(m+\gamma+1)}{m !}\left(\begin{array}{c}
\gamma-\alpha \\
k-m
\end{array}\right)\left(\begin{array}{c}
\gamma-\beta \\
k^{\prime}-m
\end{array}\right) \\
& (\operatorname{Re} \gamma>-1),
\end{aligned}
$$

yields

$$
E_{n l}^{(2)}=\frac{1}{2^{4}}\left(n-\frac{1}{2}\right)^{2}\left(5 n^{2}-5 n-3 l^{2}+3\right) Z^{-2} \frac{B^{2}}{B_{0}^{2}} \frac{e^{2}}{\left(4 \pi \epsilon_{0}\right) a_{0}}
$$

where

$$
B_{0}=\frac{\hbar}{e a_{0}^{2}}=\frac{m^{2} e^{3}}{\left(4 \pi \epsilon_{0}\right)^{2} \hbar^{3}}
$$

is the atomic unit of magnetic induction. For states with $l=n-1$ (i.e., those with $\left.n_{r}=0\right)$, the expression in eq. (30) simplifies to

$$
E_{n, n-1}^{(2)}=\frac{1}{2^{3}} n\left(n+\frac{1}{2}\right)\left(n-\frac{1}{2}\right)^{2} Z^{-2} \frac{B^{2}}{B_{0}^{2}} \frac{e^{2}}{\left(4 \pi \epsilon_{0}\right) a_{0}}
$$

\subsection{The fourth-order corrections to Coulomb energies}

Proceeding along the standard route, one finds that for the present problem the fourth-order correction to energy, $E_{n l}^{(4)}$, is given by

$$
E_{n l}^{(4)}=\int_{0}^{\infty} \mathrm{d} r P_{n l}^{(0)}(r) H^{(2)}(r) P_{n l}^{(2)}(r)
$$


where the second-order correction to the radial wave function, $P_{n l}^{(2)}(r)$, is a solution to the inhomogeneous boundaryvalue problem

$$
\begin{aligned}
& {\left[H_{l}^{(0)}(r)-E_{n}^{(0)}\right] P_{n l}^{(2)}(r)=-\left[H^{(2)}(r)-E_{n l}^{(2)}\right] P_{n l}^{(0)}(r),} \\
& P_{n l}^{(2)}(r) \stackrel{r \rightarrow 0}{\longrightarrow} 0, \quad P_{n l}^{(2)}(r) \stackrel{r \rightarrow \infty}{\longrightarrow} 0,
\end{aligned}
$$

subject to the further orthogonality restraint

$$
\int_{0}^{\infty} \mathrm{d} r P_{n l}^{(0)}(r) P_{n l}^{(2)}(r)=0 .
$$

The formal solution to the problem (34)-(35) is

$$
P_{n l}^{(2)}(r)=-\int_{0}^{\infty} \mathrm{d} r^{\prime} \widetilde{G}_{n l}^{(0)}\left(r, r^{\prime}\right)\left[H^{(2)}\left(r^{\prime}\right)-E_{n l}^{(2)}\right] P_{n l}^{(0)}\left(r^{\prime}\right),
$$

where $\widetilde{G}_{n l}^{(0)}\left(r, r^{\prime}\right)$ is a generalized (or reduced) radial Coulomb Green function associated with the Coulomb energy level $E_{n}^{(0)}$. The latter function is defined as that particular solution to the inhomogeneous boundary-value problem

$$
\begin{aligned}
& {\left[H_{l}^{(0)}(r)-E_{n}^{(0)}\right] \widetilde{G}_{n l}^{(0)}\left(r, r^{\prime}\right)=\delta\left(r-r^{\prime}\right)-P_{n l}^{(0)}(r) P_{n l}^{(0)}\left(r^{\prime}\right),} \\
& \widetilde{G}_{n l}^{(0)}\left(r, r^{\prime}\right) \stackrel{r \rightarrow 0}{\longrightarrow} 0, \quad \widetilde{G}_{n l}^{(0)}\left(r, r^{\prime}\right) \stackrel{r \rightarrow \infty}{\longrightarrow} 0,
\end{aligned}
$$

where $\delta\left(r-r^{\prime}\right)$ is the Dirac delta function, which obeys the additional orthogonality constraint

$$
\int_{0}^{\infty} \mathrm{d} r P_{n l}^{(0)}(r) \widetilde{G}_{n l}^{(0)}\left(r, r^{\prime}\right)=0 .
$$

Since the zeroth-order eigenproblem (19) is self-adjoint, the function $\widetilde{G}_{n l}^{(0)}\left(r, r^{\prime}\right)$ is symmetric in its arguments:

$$
\widetilde{G}_{n l}^{(0)}\left(r, r^{\prime}\right)=\widetilde{G}_{n l}^{(0)}\left(r^{\prime}, r\right) .
$$

When this is combined with eq. (38), one deduces the formula

$$
\int_{0}^{\infty} \mathrm{d} r^{\prime} \widetilde{G}_{n l}^{(0)}\left(r, r^{\prime}\right) P_{n l}^{(0)}\left(r^{\prime}\right)=0,
$$

which allows us to simplify eq. (36) to obtain

$$
P_{n l}^{(2)}(r)=-\int_{0}^{\infty} \mathrm{d} r^{\prime} \widetilde{G}_{n l}^{(0)}\left(r, r^{\prime}\right) H^{(2)}\left(r^{\prime}\right) P_{n l}^{(0)}\left(r^{\prime}\right) .
$$

Plugging eq. (41) into the right-hand side of eq. (33) gives the energy correction $E_{n l}^{(4)}$ in the form

$$
E_{n l}^{(4)}=-\int_{0}^{\infty} \mathrm{d} r \int_{0}^{\infty} \mathrm{d} r^{\prime} P_{n l}^{(0)}(r) H^{(2)}(r) \widetilde{G}_{n l}^{(0)}\left(r, r^{\prime}\right) H^{(2)}\left(r^{\prime}\right) P_{n l}^{(0)}\left(r^{\prime}\right)
$$

or, still more explicitly, in the form

$$
E_{n l}^{(4)}=-\left(\frac{e^{2} B^{2}}{8 m}\right)^{2} \int_{0}^{\infty} \mathrm{d} r \int_{0}^{\infty} \mathrm{d} r^{\prime} P_{n l}^{(0)}(r) r^{2} \widetilde{G}_{n l}^{(0)}\left(r, r^{\prime}\right) r^{\prime 2} P_{n l}^{(0)}\left(r^{\prime}\right)
$$

A representation of the generalized radial Coulomb Green function $\widetilde{G}_{n l}^{(0)}\left(r, r^{\prime}\right)$ which is perhaps the most suitable for the use in eq. (43) is the one in the form of a series expansion in the discrete radial Coulomb Sturmian basis. We shall construct it below.

The discrete radial Coulomb Sturmian functions are defined as solutions to the spectral problem

$$
\begin{aligned}
& {\left[-\frac{\hbar^{2}}{2 m} \frac{\mathrm{d}^{2}}{\mathrm{~d} r^{2}}+\frac{\hbar^{2}\left(l^{2}-\frac{1}{4}\right)}{2 m r^{2}}-\mu_{n_{r} l}^{(0)}(E) \frac{Z e^{2}}{\left(4 \pi \epsilon_{0}\right) r}-E\right] S_{n_{r} l}^{(0)}(E, r)=0 \quad(E<0),} \\
& S_{n_{r} l}^{(0)}(E, r) \stackrel{r \rightarrow 0}{\longrightarrow} 0, \quad S_{n_{r} l}^{(0)}(E, r) \stackrel{r \rightarrow \infty}{\longrightarrow} 0,
\end{aligned}
$$


with $E<0$ fixed and with the parameter $\mu_{n_{r} l}^{(0)}(E)$ chosen as an eigenvalue. The spectrum of this problem is purely discrete, and eigenvalues are given by

$$
\mu_{n_{r} l}^{(0)}(E)=\left(n_{r}+l+\frac{1}{2}\right) \frac{k a_{0}}{Z} \quad\left(n_{r} \in \mathbb{N}_{0}\right)
$$

where

$$
k=\sqrt{-\frac{2 m E}{\hbar^{2}}}
$$

Eigenfunctions, orthonormal in the sense of

$$
\int_{0}^{\infty} \mathrm{d} r \frac{Z e^{2}}{\left(4 \pi \epsilon_{0}\right) r} S_{n_{r} l}^{(0)}(E, r) S_{n_{r}^{\prime} l}^{(0)}(E, r)=\delta_{n_{r} n_{r}^{\prime}}
$$

are

$$
S_{n_{r} l}^{(0)}(E, r)=\sqrt{\frac{\left(4 \pi \epsilon_{0}\right) n_{r} !}{Z e^{2}\left(n_{r}+2 l\right) !}}(2 k r)^{l+1 / 2} \mathrm{e}^{-k r} L_{n_{r}}^{(2 l)}(2 k r) .
$$

Contrary to the discrete Coulomb eigenfunctions (24), the Sturmians (48) form a complete set, the corresponding closure relation being

$$
\frac{Z e^{2}}{\left(4 \pi \epsilon_{0}\right) r} \sum_{n_{r}=0}^{\infty} S_{n_{r} l}^{(0)}(E, r) S_{n_{r} l}^{(0)}\left(E, r^{\prime}\right)=\delta\left(r-r^{\prime}\right) .
$$

If the parameter $E$ coincides with the Coulomb energy eigenvalue $E_{n}^{(0)}$ displayed in eq. (20) (we assume $n$ is related to $n_{r}$ and $l$ used here as in eq. (9)), it is easy to see from eqs. (45), (46), (20) and (21) that one has

$$
\mu_{n_{r} l}^{(0)}\left(E_{n}^{(0)}\right)=1 \quad\left(n=n_{r}+l+1\right)
$$

Similarly, from eqs. (48), (44), (20), (21) and (25) one infers the relationship

$$
S_{n_{r} l}^{(0)}\left(E_{n}^{(0)}, r\right)=\frac{N_{n}}{Z} \sqrt{\frac{\left(4 \pi \epsilon_{0}\right) a_{0}}{e^{2}}} P_{n l}^{(0)}(r) \quad\left(n=n_{r}+l+1\right) .
$$

The radial Coulomb Green function, $G_{l}^{(0)}\left(E, r, r^{\prime}\right)$, is defined to be a solution to the inhomogeneous equation

$$
\left[H_{l}^{(0)}(r)-E\right] G_{l}^{(0)}\left(E, r, r^{\prime}\right)=\delta\left(r-r^{\prime}\right) \quad(E<0)
$$

subject to the boundary constraints

$$
G_{l}^{(0)}\left(E, r, r^{\prime}\right) \stackrel{r \rightarrow 0}{\longrightarrow} 0, \quad G_{l}^{(0)}\left(E, r, r^{\prime}\right) \stackrel{r \rightarrow \infty}{\longrightarrow} 0 .
$$

Since the Sturmian functions (48) form a complete set, the Green function $G_{l}^{(0)}\left(E, r, r^{\prime}\right)$ may be sought in the form of the series

$$
G_{l}^{(0)}\left(E, r, r^{\prime}\right)=\sum_{n_{r}=0}^{\infty} C_{n_{r} l}^{(0)}\left(E, r^{\prime}\right) S_{n_{r} l}^{(0)}(E, r)
$$

To determine the expansion coefficients $C_{n_{r} l}^{(0)}(E, r)$, we plug eq. (53) into eq. (52a), multiply both sides of the resulting identity by $S_{n_{r}^{\prime} l}^{(0)}(E, r)$, then integrate with respect to $r$ over the interval $[0, \infty)$, and apply the orthogonality relation (48). Upon the replacement of $n_{r}^{\prime}$ with $n_{r}$, this yields

$$
C_{n_{r} l}^{(0)}\left(E, r^{\prime}\right)=\frac{1}{\mu_{n_{r} l}^{(0)}(E)-1} S_{n_{r} l}^{(0)}\left(E, r^{\prime}\right)
$$

hence, we obtain the following symmetric Sturmian expansion of $G_{l}^{(0)}\left(E, r, r^{\prime}\right)$ :

$$
G_{l}^{(0)}\left(E, r, r^{\prime}\right)=\sum_{n_{r}=0}^{\infty} \frac{S_{n_{r} l}^{(0)}(E, r) S_{n_{r} l}^{(0)}\left(E, r^{\prime}\right)}{\mu_{n_{r} l}^{(0)}(E)-1}
$$


It follows, from eqs. (37), (38) and (52), that the generalized radial Coulomb Green function $\widetilde{G}_{n l}^{(0)}\left(r, r^{\prime}\right)$ may be obtained from the radial Coulomb Green function $G_{l}^{(0)}\left(E, r, r^{\prime}\right)$ through the limit procedure

$$
\widetilde{G}_{n l}^{(0)}\left(r, r^{\prime}\right)=\lim _{E \rightarrow E_{n}^{(0)}}\left[G_{l}^{(0)}\left(E, r, r^{\prime}\right)-\frac{P_{n l}^{(0)}(r) P_{n l}^{(0)}\left(r^{\prime}\right)}{E_{n}^{(0)}-E}\right] .
$$

By virtue of the de l'Hospital rule, the latter equation is equivalent to the following one:

$$
\widetilde{G}_{n l}^{(0)}\left(r, r^{\prime}\right)=\lim _{E \rightarrow E_{n}^{(0)}} \frac{\partial}{\partial E}\left[\left(E-E_{n}^{(0)}\right) G_{l}^{(0)}\left(E, r, r^{\prime}\right)\right],
$$

which is particularly suitable for the construction of the Sturmian expansion of $\widetilde{G}_{n l}^{(0)}\left(r, r^{\prime}\right)$. Inserting the series representation (55) into the right-hand side of eq. (57) and then making use of the relationships

$$
\begin{aligned}
\frac{\partial S_{n_{r} l}^{(0)}(E, r)}{\partial E} & =\frac{r}{2 E} \frac{\mathrm{d} S_{n_{r} l}^{(0)}(E, r)}{\mathrm{d} r}, \\
\frac{E-E_{n}^{(0)}}{\mu_{n_{r} l}^{(0)}(E)-1} & =E_{n}^{(0)}\left[\mu_{n_{r} l}^{(0)}(E)+1\right], \\
\lim _{E \rightarrow E_{n}^{(0)}} \frac{E-E_{n}^{(0)}}{\mu_{n_{r} l}^{(0)}(E)-1} & =2 E_{n}^{(0)} \quad\left(n=n_{r}+l+1\right), \\
\lim _{E \rightarrow E_{n}^{(0)}} \frac{\partial}{\partial E} \frac{E-E_{n}^{(0)}}{\mu_{n_{r} l}^{(0)}(E)-1} & =\frac{1}{2} \quad\left(n=n_{r}+l+1\right), \\
\mu_{n_{r}^{\prime} l}^{(0)}\left(E_{n}^{(0)}\right) & =\frac{n_{r}^{\prime}+l+\frac{1}{2}}{N_{n}},
\end{aligned}
$$

which may be easily derived from the defining eqs. (45) and (48), one eventually arrives at the sought Sturmian expansion of the generalized radial Coulomb Green function, which is

$$
\begin{aligned}
\widetilde{G}_{n l}^{(0)}\left(r, r^{\prime}\right)= & N_{n} \sum_{\substack{n_{r}^{\prime}=0 \\
\left(n_{r}^{\prime} \neq n_{r}\right)}}^{\infty} \frac{S_{n_{r}^{\prime} l}^{(0)}\left(E_{n}^{(0)}, r\right) S_{n_{r}^{\prime} l}^{(0)}\left(E_{n}^{(0)}, r^{\prime}\right)}{n_{r}^{\prime}-n_{r}}+\frac{1}{2} S_{n_{r} l}^{(0)}\left(E_{n}^{(0)}, r\right) S_{n_{r} l}^{(0)}\left(E_{n}^{(0)}, r^{\prime}\right) \\
& +r \frac{\mathrm{d} S_{n_{r} l}^{(0)}\left(E_{n}^{(0)}, r\right)}{\mathrm{d} r} S_{n_{r} l}^{(0)}\left(E_{n}^{(0)}, r^{\prime}\right)+S_{n_{r} l}^{(0)}\left(E_{n}^{(0)}, r\right) r^{\prime} \frac{\mathrm{d} S_{n_{r} l}^{(0)}\left(E_{n}^{(0)}, r^{\prime}\right)}{\mathrm{d} r^{\prime}} \quad\left(n_{r}=n-l-1\right) .
\end{aligned}
$$

Once the Sturmian expansion of $\widetilde{G}_{n l}^{(0)}\left(r, r^{\prime}\right)$ has been found, we are ready to complete the task to find the fourthorder energy correction $E_{n l}^{(4)}$. To this end, we insert eq. (63) into eq. (43) and use the relationship in eq. (51), together with integrations by parts, to eliminate derivatives of Sturmian functions. This gives $E_{n l}^{(4)}$ in the form

$$
\begin{aligned}
E_{n l}^{(4)}= & -\left(\frac{e^{2} B^{2}}{8 m}\right)^{2}\left\{N_{n} \sum_{\substack{n_{r}^{\prime}=0 \\
\left(n_{r}^{\prime} \neq n_{r}\right)}}^{\infty} \frac{\left[\int_{0}^{\infty} \mathrm{d} r r^{2} P_{n l}^{(0)}(r) S_{n_{r}^{\prime} l}^{(0)}\left(E_{n}^{(0)}, r\right)\right]^{2}}{n_{r}^{\prime}-n_{r}}-\frac{5}{2}\left[\int_{0}^{\infty} \mathrm{d} r r^{2} P_{n l}^{(0)}(r) S_{n_{r} l}^{(0)}\left(E_{n}^{(0)}, r\right)\right]^{2}\right\} \\
& \left(n_{r}=n-l-1\right) .
\end{aligned}
$$

The integrals in eq. (64) may be taken after one exploits eqs. (25) and (48), with the use of the integration formula

$$
\begin{aligned}
& \int_{0}^{\infty} \mathrm{d} x x^{\alpha+3} \mathrm{e}^{-x} L_{k}^{(\alpha)}(x) L_{k^{\prime}}^{(\alpha)}(x)=-\frac{\Gamma(k+\alpha+1)}{(k-3) !} \delta_{k^{\prime}, k-3}+3(2 k+\alpha-1) \frac{\Gamma(k+\alpha+1)}{(k-2) !} \delta_{k^{\prime}, k-2} \\
& \quad-3\left(5 k^{2}+5 \alpha k+\alpha^{2}+1\right) \frac{\Gamma(k+\alpha+1)}{(k-1) !} \delta_{k^{\prime}, k-1}+(2 k+\alpha+1)\left(10 k^{2}+10 k+10 \alpha k+\alpha^{2}+5 \alpha+6\right) \frac{\Gamma(k+\alpha+1)}{k !} \delta_{k^{\prime} k} \\
& \quad-3\left(5 k^{2}+10 k+5 \alpha k+\alpha^{2}+5 \alpha+6\right) \frac{\Gamma(k+\alpha+2)}{k !} \delta_{k^{\prime}, k+1}+3(2 k+\alpha+3) \frac{\Gamma(k+\alpha+3)}{k !} \delta_{k^{\prime}, k+2} \\
& \quad-\frac{\Gamma(k+\alpha+4)}{k !} \delta_{k^{\prime}, k+3} \quad(\operatorname{Re} \alpha>-4),
\end{aligned}
$$


which generalizes the one in eq. (28) and, similarly to the latter, may be derived from the general expression (29). Since only terms with $n_{r}^{\prime}$ constrained by $1 \leqslant\left|n_{r}^{\prime}-n_{r}\right| \leqslant 3$ are seen to contribute non-vanishingly to the sum in eq. (64), we eventually obtain

$$
E_{n l}^{(4)}=-\frac{1}{2^{10}}\left(n-\frac{1}{2}\right)^{6}\left(143 n^{4}-286 n^{3}-90 n^{2} l^{2}+582 n^{2}+90 n l^{2}-439 n-21 l^{4}-138 l^{2}+159\right) Z^{-6} \frac{B^{4}}{B_{0}^{4}} \frac{e^{2}}{\left(4 \pi \epsilon_{0}\right) a_{0}} .
$$

For states with $l=n-1$ (i.e., those with $n_{r}=0$ ), eq. (66) becomes

$$
E_{n, n-1}^{(4)}=-\frac{1}{2^{9}} n\left(n+\frac{1}{2}\right)\left(n-\frac{1}{2}\right)^{6}\left(16 n^{2}+26 n+11\right) Z^{-6} \frac{B^{4}}{B_{0}^{4}} \frac{e^{2}}{\left(4 \pi \epsilon_{0}\right) a_{0}} .
$$

For the ground state $(n=1)$, eq. (67) yields

$$
E_{10}^{(4)}=-\frac{159}{65536} Z^{-6} \frac{B^{4}}{B_{0}^{4}} \frac{e^{2}}{\left(4 \pi \epsilon_{0}\right) a_{0}}
$$

This differs from the result announced in refs. [25] (eq. (32)) and [31] (eq. (6.59)), which is

$$
E_{10}^{(4)}=-\frac{153}{65536} Z^{-6} \frac{B^{4}}{B_{0}^{4}} \frac{e^{2}}{\left(4 \pi \epsilon_{0}\right) a_{0}} .
$$

The latter one is thus found to be incorrect.

\section{Summary and concluding remarks}

On the preceding pages, we have shown that energy levels of the planar hydrogen-like atom placed in a weak, static, uniform magnetic field of induction $\boldsymbol{B}$ perpendicular to the atomic plane may be expressed in the form

$$
E_{n l m_{l}}=E_{n}^{(0)}+E_{m_{l}}^{(1)}+E_{n l}^{(2)}+E_{n l}^{(4)}+O\left(Z^{-10}\left(B / B_{0}\right)^{6}\right)
$$

where

$$
E_{\ldots}^{(k)}=\varepsilon_{\ldots}^{(k)} Z^{-2 k+2} \frac{B^{k}}{B_{0}^{k}} \frac{e^{2}}{\left(4 \pi \epsilon_{0}\right) a_{0}} .
$$

In eq. (71), $Z$ is an electric charge of the atomic nucleus in units of the elementary charge $e, a_{0}$ is the Bohr radius,

$$
B_{0}=\frac{m^{2} e^{3}}{\left(4 \pi \epsilon_{0}\right)^{2} \hbar^{3}} \simeq 2.35 \times 10^{5} \mathrm{~T}
$$

is the atomic unit of magnetic induction, while the dimensionless and $Z$-independent coefficients $\varepsilon_{\ldots}^{(k)}$ are given by

$$
\begin{aligned}
& \varepsilon_{n}^{(0)}=-\frac{1}{2\left(n-\frac{1}{2}\right)^{2}}, \\
& \varepsilon_{m_{l}}^{(1)}=\frac{1}{2} m_{l} \\
& \varepsilon_{n l}^{(2)}=\frac{1}{2^{4}}\left(n-\frac{1}{2}\right)^{2}\left(5 n^{2}-5 n-3 l^{2}+3\right)
\end{aligned}
$$

and

$$
\varepsilon_{n l}^{(4)}=-\frac{1}{2^{10}}\left(n-\frac{1}{2}\right)^{6}\left(143 n^{4}-286 n^{3}-90 n^{2} l^{2}+582 n^{2}+90 n l^{2}-439 n-21 l^{4}-138 l^{2}+159\right),
$$

with $n \in \mathbb{N}_{+}, m_{l} \in \mathbb{Z}$ and $0 \leqslant l=\left|m_{l}\right| \leqslant n-1$. Numerical values of the coefficients $\varepsilon_{n l}^{(2)}$ and $\varepsilon_{n l}^{(4)}$ for states with $1 \leqslant n \leqslant 4$ are displayed in table 1 .

It has to be emphasized that the formula in eq. (70) is valid only if the electron spin is ignored. If this cannot be done, the Schrödinger equation (1) should be replaced with the planar Pauli equation

$$
\left\{\frac{\left\{\boldsymbol{\sigma} \cdot[-\mathrm{i} \hbar \boldsymbol{\nabla}+e \boldsymbol{A}(\boldsymbol{r})]^{2}\right.}{2 m}-\frac{Z e^{2}}{\left(4 \pi \epsilon_{0}\right) r}\right\} \Psi(\boldsymbol{r})=E \Psi(\boldsymbol{r}) \quad\left(\boldsymbol{r} \in \mathbb{R}^{2}\right)
$$


Table 1. Numerical values of the coefficients $\varepsilon_{n l}^{(2)}$ and $\varepsilon_{n l}^{(4)}$, defined in eqs. (75) and (76), for $1 \leqslant n \leqslant 4$ and $0 \leqslant l \leqslant n-1$.

\begin{tabular}{|c|c|c|c|c|c|}
\hline \multirow[t]{2}{*}{$n$} & \multirow[t]{2}{*}{$l$} & \multicolumn{2}{|c|}{$\varepsilon_{n l}^{(2)}$} & \multicolumn{2}{|c|}{$\varepsilon_{n l}^{(4)}$} \\
\hline & & Rational form & Factorized form & Rational form & Factorized form \\
\hline \multirow{3}{*}{1} & \multirow{2}{*}{0} & 3 & 3 & 159 & $3 \times 53$ \\
\hline & & $\overline{64}$ & $\overline{2^{6}}$ & $\overline{65536}$ & $-\frac{2^{16}}{2}$ \\
\hline & \multirow{2}{*}{0} & 117 & $3^{2} \times 13$ & 1172961 & $3^{6} \times 1609$ \\
\hline \multirow{3}{*}{2} & & $\overline{64}$ & $2^{6}$ & $\overline{65536}$ & $2^{16}$ \\
\hline & \multirow{2}{*}{1} & 45 & $3^{2} \times 5$ & 462915 & $3^{6} \times 5 \times 127$ \\
\hline & & $\overline{32}$ & $\overline{2^{5}}$ & $-\overline{32768}$ & $2^{15}$ \\
\hline \multirow{7}{*}{3} & \multirow{2}{*}{0} & 825 & $3 \times 5^{2} \times 11$ & 124078125 & $3 \times 5^{6} \times 2647$ \\
\hline & & $\overline{64}$ & $2^{6}$ & 65536 & $2^{16}$ \\
\hline & \multirow{2}{*}{1} & 375 & $3 \times 5^{3}$ & 56578125 & $3 \times 5^{6} \times 17 \times 71$ \\
\hline & & $\overline{32}$ & $\overline{2^{5}}$ & $\overline{32768}$ & $2^{15}$ \\
\hline & 2 & $\underline{525}$ & $3 \times 5^{2} \times 7$ & 76453125 & $3 \times 5^{6} \times 7 \times 233$ \\
\hline & 2 & $\overline{64}$ & $2^{6}$ & 65536 & $2^{16}$ \\
\hline & \multirow{2}{*}{0} & 3087 & $3^{2} \times 7^{3}$ & 3061109331 & $3^{2} \times 7^{8} \times 59$ \\
\hline \multirow{7}{*}{4} & & 64 & $2^{6}$ & 65536 & $2^{16}$ \\
\hline & \multirow{2}{*}{1} & 735 & $3 \times 5 \times 7^{2}$ & 728835555 & $3 \times 5 \times 7^{7} \times 59$ \\
\hline & & $\overline{16}$ & $\frac{2^{4}}{}$ & 16384 & $2^{14}$ \\
\hline & \multirow{2}{*}{2} & 2499 & $3 \times 7^{2} \times 17$ & 2448393339 & $3 \times 7^{7} \times 991$ \\
\hline & & 64 & $2^{6}$ & 65536 & $2^{16}$ \\
\hline & \multirow{2}{*}{3} & 441 & $3^{2} \times 7^{2}$ & 392830011 & $3^{2} \times 7^{7} \times 53$ \\
\hline & & $\overline{16}$ & $2^{4}$ & 16384 & $2^{14}$ \\
\hline
\end{tabular}

where $\boldsymbol{\sigma}=\left(\sigma_{x}, \sigma_{y}\right)$ is the two-dimensional Pauli matrix vector, and $\Psi(\boldsymbol{r})$ is a two-component Pauli spinor. Equation (77) may be cast into the form

$$
\left\{\frac{[-\mathrm{i} \hbar \boldsymbol{\nabla}+e \boldsymbol{A}(\boldsymbol{r})]^{2}}{2 m}+\frac{e \hbar B}{m} \Sigma_{z}-\frac{Z e^{2}}{\left(4 \pi \epsilon_{0}\right) r}\right\} \Psi(\boldsymbol{r})=E \Psi(\boldsymbol{r}),
$$

with

$$
\Sigma_{z}=\frac{1}{2} \sigma_{z}
$$

where $\sigma_{z}$ is the third Pauli matrix. It is then evident that eq. (78), supplemented with the regularity constraints on $\Psi(\boldsymbol{r})$ analogous to those introduced under eq. (1), possesses separated eigenfunctions of the form

$$
\Psi_{n l m_{l} m_{s}}(r, \varphi)=\frac{1}{\sqrt{r}} P_{n l}(r) \frac{\mathrm{e}^{\mathrm{i} m_{l} \varphi}}{\sqrt{2 \pi}} \chi_{m_{s}}
$$

where $P_{n l}(r)$ is the same radial function which has appeared in the preceding sections, while $\chi_{m_{s}}$ is the spin one-half eigenfunction obeying

$$
\Sigma_{z} \chi_{m_{s}}=m_{s} \chi_{m_{s}} \quad\left(m_{s}= \pm \frac{1}{2}\right)
$$

and that the energy spectrum is of the form

$$
E_{n l m_{l} m_{s}}=E_{n}^{(0)}+E_{m_{l} m_{s}}^{(1)}+E_{n l}^{(2)}+E_{n l}^{(4)}+O\left(Z^{-10}\left(B / B_{0}\right)^{6}\right)
$$

with the terms $E_{n}^{(0)}, E_{n l}^{(2)}$ and $E_{n l}^{(4)}$ being identical to those derived before, and with

$$
E_{m_{l} m_{s}}^{(1)}=\frac{1}{2}\left(m_{l}+2 m_{s}\right) \frac{B}{B_{0}} \frac{e^{2}}{\left(4 \pi \epsilon_{0}\right) a_{0}} \quad\left(=\left(m_{l}+2 m_{s}\right) \frac{e \hbar B}{2 m}\right) .
$$

There are two natural directions in which the analysis presented in this paper might be extended. First, one might investigate the impact of the magnetic field on energy levels of a planar two-center Coulomb problem [41]. The second challenge would be to consider perturbatively, but still analytically, a planar one-center system analogous to the one studied above, but now with special relativity taken into account through the use of the Dirac, rather than the Schrödinger or Pauli, equation. We are currently working on the latter problem. 
Open Access This is an open access article distributed under the terms of the Creative Commons Attribution License (http://creativecommons.org/licenses/by/4.0), which permits unrestricted use, distribution, and reproduction in any medium, provided the original work is properly cited.

\section{References}

1. O. Akimoto, H. Hasegawa, J. Phys. Soc. Jpn. 22, 181 (1967).

2. L.P. Gor'kov, I.E. Dzyaloshinskii, Sov. Phys. JETP 26, 449 (1968).

3. M. Shinada, K. Tanaka, J. Phys. Soc. Jpn. 29, 1258 (1970).

4. A.H. MacDonald, D.S. Ritchie, Phys. Rev. B 33, 8336 (1986).

5. B.G. Adams, Theor. Chim. Acta 73, 459 (1988).

6. W. Edelstein, H.N. Spector, R. Marasas, Phys. Rev. B 39, 7697 (1989).

7. J.-L. Zhu, Y. Cheng, J.-J. Xiong, Phys. Lett. A 145, 358 (1990).

8. J.-L. Zhu, Y. Cheng, J.-J. Xiong, Phys. Rev. B 41, 10792 (1990).

9. P. Martín, J.J. Rodriguez-Nuñez, J.L. Marquez, Phys. Rev. B 45, 8359 (1992).

10. V.-H. Le, T.-G. Nguyen, J. Phys. A 26, 1409 (1993).

11. H. Lehmann, N.H. March, Pure Appl. Chem. 67, 457 (1995).

12. M. Taut, J. Phys. A 28, 2081 (1995).

13. V.M. Villalba, R. Pino, Phys. Scr. 58, 605 (1998).

14. V.M. Villalba, R. Pino, Phys. Lett. A 238, 49 (1998).

15. C.-L. Ho, V.R. Khalilov, Phys. Rev. A 61, 032104 (2000).

16. V.M. Villalba, R. Pino, Physica E 10, 561 (2001).

17. M. Robnik, V.G. Romanovski, J. Phys. A 36, 7923 (2003).

18. V.M. Villalba, R. Pino, Mod. Phys. Lett. B 17, 1331 (2003).

19. A. Soylu, O. Bayrak, I. Boztosun, Int. J. Mod. Phys. E 15, 1263 (2006).

20. A. Soylu, I. Boztosun, Physica B 396, 150 (2007).

21. A. Rutkowski, A. Poszwa, Phys. Scr. 79, 065010 (2009).

22. A. Poszwa, A. Rutkowski, Acta Phys. Pol. A 117, 439 (2010).

23. M. Gadella, J. Negro, L.M. Nieto, G.P. Pronko, Int. J. Theor. Phys. 50, 2019 (2011).

24. A. Poszwa, Phys. Scr. 84, 055002 (2011).

25. N.-T. Hoang-Do, V.-H. Hoang, V.-H. Le, J. Math. Phys. 54, 052105 (2013).

26. N.-T. Hoang-Do, D.-L. Pham, V.-H. Le, Physica B 423, 31 (2013).

27. A. Poszwa, Phys. Scr. 89, 065401 (2014).

28. M.A. Escobar, A.V. Turbiner, Ann. Phys. 340, 37 (2014).

29. M.A. Escobar, A.V. Turbiner, Ann. Phys. 359, 405 (2015).

30. M.A. Escobar-Ruiz, Ann. Phys. 351, 714 (2014).

31. I. Feranchuk, A. Ivanov, V.-H. Le, A. Ulyanenkov, Non-perturbative description of quantum systems, Lect. Notes Phys., Vol. 894 (Springer, 2015) chapt. 6.

32. C. Flavio, C. Enrique, M. Pablo, C.-V. Luis, J. Phys.: Conf. Ser. 574, 012105 (2015.)

33. L. Liu, Q. Hao, Theor. Math. Phys. 183, 730 (2015).

34. J.S. Ardenghi, M. Gadella, J. Negro, Phys. Lett. A 380, 1817 (2016).

35. N.-T.D. Hoang, D.-A.P. Nguyen, V.-H. Hoang, V.-H. Le, Physica B 495, 16 (2016).

36. D.-N. Le, N.-T.D. Hoang, V.-H. Le, J. Math. Phys. 58, 042102 (2017).

37. F.M. Fernández, Phys. Rev. A 45, 1333 (1992).

38. B.G. Adams, Phys. Rev. A 46, 4060 (1992).

39. W. Magnus, F. Oberhettinger, R.P. Soni, Formulas and Theorems for the Special Functions of Mathematical Physics, 3rd edition (Springer, Berlin, 1966).

40. R. Szmytkowski, J. Phys. B 30, 825 (1997) 30, 2747(E) (1997).

41. M.A. González León, J. Mateos Guilarte, M. de la Torre Mayado, EPL 114, 30007 (2016). 